\title{
60GHZ-BAND FLIP-CHIP MMIC MODULES FOR IEEE1394 WIRELESS ADAPTERS
}

\author{
K. Maruhashi, M. Ito, K. Ikuina, T. Hashiguchi, J. Matsuda, W. Domon, S. Iwanaga*, \\ N. Takahashi**, T. Ishihara**, Y. Yoshida**, I. Izumi** and K. Ohata \\ NEC Corporation, 2-9-1 Seiran, Otsu, Shiga 520-0833, JAPAN, e-mail:k-maruhashi@bl.jp.nec.com \\ *NEC Kansai, Ltd., 2-9-1 Seiran, Otsu, Shiga 520-0833, JAPAN \\ **NEC Engineering, Ltd., 1753 Shimonumabe, Nakahara-ku, Kawasaki, Kanagawa 211-0011, JAPAN
}

\begin{abstract}
60GHz-band 500-Mpbs transmitter and receiver multi-chip modules (MCMs) are presented, in which MMICs and a filter are mounted using flip-chip bonding technique. A multi-layer Low Temperature Cofired Ceramic (LTCC) substrate is adopted for a package. The MCMs are directly bonded with printed wiring boards using ball grid array technique, achieving connections for signals and biasing. A developed 500-Mbps ASK transceiver exhibits an average output power of $10 \mathrm{dBm}$ and a minimum received power of $55 \mathrm{dBm}$. The transceiver is applied to the IEEE1394 wireless adapter which demonstrates 17-m communication distance in line of sight.
\end{abstract}

\section{INTRODUCTION}

Increasing demands for multimedia data links stimulate the development of low-cost wireless transceivers with a bit-rate more than 100Mbps. A $60-\mathrm{GHz}$ unlicensed frequency band (Japan/US) having the advantage of wide-bandwidth $(5-7 \mathrm{GHz})$ availability facilitates the realization of high-speed data transmission systems. We have recently proposed and demonstrated the first IEEE1394 S400 (400Mbps) wireless adapters to confirm system architecture proof-of-concept (1)-(3). The ASK modulation scheme was adopted to achieve 500-Mbps data transmission required for the transceiver. The ASK transceiver has the advantage of simple configuration with direct modulation/demodulation, which does not require a high-linearity amplifier and an oscillator with low phase noise. This results in low cost. The MCMs in the previous transceiver utilized microstrip-line-type MMICs with wire bonding and external waveguide filters, which was not suitable for mass production. The purpose of this work is to develop low-cost flip-chip MMIC modules. Application of flip-chip technology to millimeter-wave modules offers excellent reproducibility and performance as well as reduction in fabrication steps. Several flip-chip MMIC modules (4)-(6) have been developed. However, they have not included filters. In this paper, we demonstrate integration of a flip-chip filter with coplanar-type MMICs for the first time. The developed MCMs are applied to compact IEEE1394 wireless adapters.

\section{TRANSCEIVER DIAGRAM}

A block diagram of the transmitter (TX) and the receiver (RX) is shown in Fig. 1. TX is composed of a frequency doubler, a modulator and a medium power amplifier (MPA). A filter is not necessary because occupied frequency bandwidth for a 500 -Mbps ASK-modulated signal is less than $2.5 \mathrm{GHz}$ which is permitted by the Japanese technical regulation for $60-\mathrm{GHz}$ small power systems. On the other hand, RX is composed of a low noise amplifier (LNA), a filter and a detector. Two carrier frequencies of 60.05- and 63$\mathrm{GHz}$ are used to achieve full duplex links. To reduce internal signal leakage from TX to RX via antennas in a set of the transceiver, the filter in RX is indispensable.

\section{MMIC AND FILTER PERFORMANCES}

A coplanar-type MMIC chipset used in the modules has been reported except the frequency doubler (7). The MPA shows a saturated output power of $14 \mathrm{dBm}$ with $12-\mathrm{dB}$ linear gain. The ASK modulator exhibits an onoff ratio better than $18 \mathrm{~dB}$. A $30 / 60-\mathrm{GHz}$ frequency doubler shows a saturated output power of $0 \mathrm{dBm}$. The LNA exhibits $20-\mathrm{dB}$ gain with an NF of $4 \mathrm{~dB}$. The detector shows a minimum detection power of $-32 \mathrm{dBm}$ 
defined at 10-mV output voltage. All MMICs operate under 3.3-V operation. A planar dielectric waveguide filter has been developed for this work (8). CPW I/O ports directly formed on the filter enable flip-chip mounting on the module. The filter exhibits $2.8-\mathrm{dB}$ insertion loss and a rejection more than $20 \mathrm{~dB}$ at $3-\mathrm{GHz}$ separation from a center frequency.

\section{MODULE TECHNOLOGY}

A schematic cross-sectional view of the MCM is shown in Fig. 2. We employ the multi-layer LTCC substrate as package structure, featuring cavities for flip-chip mounting, low-dispersion embedded coplanar waveguide (E-CPW) for thick-film printing and low-loss feedthrough structure (6)(9). Baseband signals, 30$\mathrm{GHz} \mathrm{CW}$ signals and dc power are fed via solder balls from a printed wiring board (PWB). A CPW to waveguide transition is formed on the substrate for antenna connection. Chip capacitors inside the MCM are eliminated, and instead of them, capacitors on the backside of the PWB are effective for bias de-coupling. The fabricated TX- and RX-MCMs are shown in Fig. 3. The fabrication process of the package does not include thin-film printing using lithography technique and it is completed just after co-firing and gold plating. Therefore, the number of components and the number of fabrication steps are reduced. The MMICs and the filter are mounted on the package with gold stud bumps by using a thermal compression method.

\section{TRANSCEIVER \& IEEE1394 WIRELESS ADAPTER}

The measured average output power of TX under 500-Mbps ASK operation with 50\%-duty is shown in Fig. 4. The output power of $10 \mathrm{dBm}$ was obtained at a $30-\mathrm{GHz}$ input power of $-5 \mathrm{dBm}$. The $99 \%$-power bandwidth of the output spectrum was measured to be about $1.6 \mathrm{GHz}$. The RX frequency response of sensitivity is shown in Fig.5. The frequency response was similar to that for the filter insertion loss, indicating successful integration of the filter. The rejection of internal signal leakage from TX in a set of the transceiver can be improved by more than 20dB. A good eye diagram of the demodulated signal was observed as shown in Fig. 6. The measured bit-error-rate performance of RX is shown in Fig. 7. The minimum received power defined at a bit-error rate of $10 \mathrm{E}-12$ was $-55 \mathrm{dBm}$.

The developed IEEE1394 wireless adapter is shown in Fig.8. The adapter consists of TX, RX, and 1394 long-haul PHY and can support S400 that is maximum data rate of IEEE1394a-2000. The size of the adapter is $141 \mathrm{~mm} \times 91 \mathrm{~mm} \times 32 \mathrm{~mm}$ and the volume is one-fifth of that for the previous adapter (1)(2). According to the technical regulation in Japan, the average output power was reduced to be $7 \mathrm{dBm}$ (10-dBm peak power) by adjusting bias voltages to the MMICs. The gain of the employed planar antennas was $\sim 18 \mathrm{dBi}$. In the experiment transmitting DV formatted video data, error-free transmission distance was up to $17 \mathrm{~m}$ in line of sight.

\section{CONCLUSION}

We have successfully integrated flip-chip MMICs and a filter in the multi-layer LTCC package. For cost reduction, the numbers of components and the number of fabrication steps were reduced. The developed MCMs were applied to the IEEE1394 wireless adapter and showed excellent performance for high-speed short-range networking systems.

\section{ACKNOWLEDGEMENT}

The authors would thank K. Yamanoguchi, N. Samoto for their useful discussions. Support from T. Sugimoto, S. Yamazaki, Y. Shimada, M. Kuzuhara, I. Mito, T. Uji, M. Ogawa and H. Mizuta is appreciated.

\section{REFERENCES}

(1) K. Ohata, K. Maruhashi, J. Matsuda, T. Ishihara, N. Takahashi and I. Izumi, "Ultra High Speed 60GHz-Band ASK Transceiver," Topical Symposium on Millimeter Waves, Digest, pp.173-176, Yokosuka, Japan, March, 2000.

(2) K. Ohata, K. Maruhashi, J. Matsuda, M. Ito, W. Domon and S. Yamazaki, ”A 500Mbps 60GHz-band Transceiver for IEEE 1394 Wireless Home Networks," 30th European Microwave Conference, Digest, Vol.1, pp.289-292, Paris, Oct., 2000. 
(3) J. Matsuda, W. Domon, K. Ohata, K. Maruhashi, M. Ito and S. Yamazaki, "Proposal of 1394 Wireless Home Network using Millimeter-Wave Technologies", WPMC '00 Conference Proceedings, Vol.1, pp.238-242, Bangkok, Nov. 2000.

(4) T. v. Kerssenbrock and P. Heide, "Novel 77 GHz Flip-chip Sensor Modules for Automotive Radar Applications," IEEE MTT-S International Microwave Symposium, Digest, pp.289-292, Anaheim, June, 1999.

(5) Y. Ohashi, T. Shimura, Y. Kawasaki, S. Aoki, H. Someta, T. Shimura, and T. Hirose, "76 GHz Flip-chip MMICs in Through-hole Packages," 28th European Microwave Conference, Digest, Vol.2, pp.433-438, Amsterdam, Oct., 1998.

(6) K. Maruhashi, M. Ito, L. Desclos, K. Ikuina, N. Senba, N. Takahashi and K. Ohata, "Low-cost Antenna-integrated 60GHz-band Transmitter/Receiver Modules Utilizing Multi-layer Low-temperature Co-fired Ceramic Technology,” IEEE International Solid-state Circuits Symposium, Digest, pp.324-325, San Francisco, Feb., 2000.

(7) K. Maruhashi, M. Ito and K. Ohata, "A 60GHz-band Coplanar-MMIC Chipset for 500Mbps ASK Transceivers," 22nd GaAs IC Symposium, Digest, pp.179-182, Seattle, Nov., 2000.

(8) M. Ito, K. Maruhashi, K. Ikuina, T. Hashiguchi, S. Iwanaga and K. Ohata, "A 60GHz-band Planar Dielectric Waveguide Filter for Flip-chip Modules,” IEEE MTT-S International Microwave Symposium, Digest, pp.15971600, Phoenix, May, 2001.

(9) M. Ito, K. Maruhashi, K. Ikuina, N. Senba, N. Takahashi and K. Ohata, "Low cost Multi-layer Ceramic Package for Flip-chip MMIC up to W-Band," IEEE MTT-S International Microwave Symposium, Digest, pp.57-60, Boston, June, 2000.
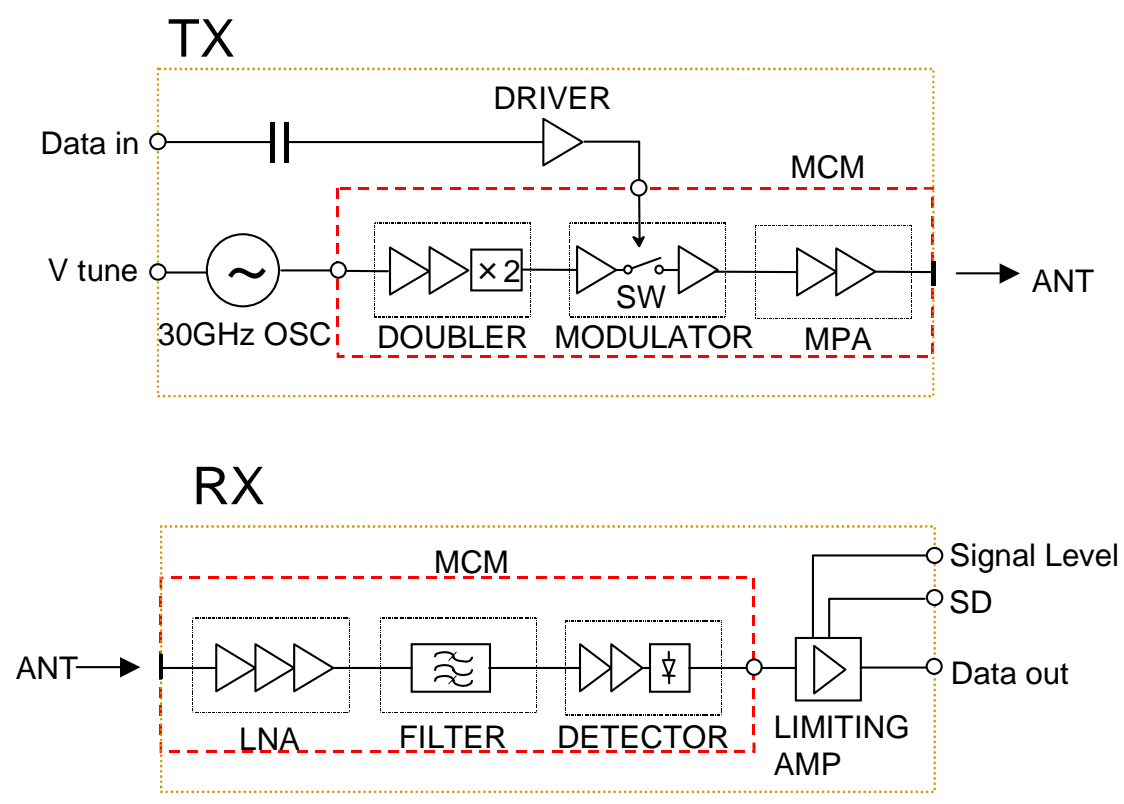

Fig. 1 Block diagram of the transceiver.

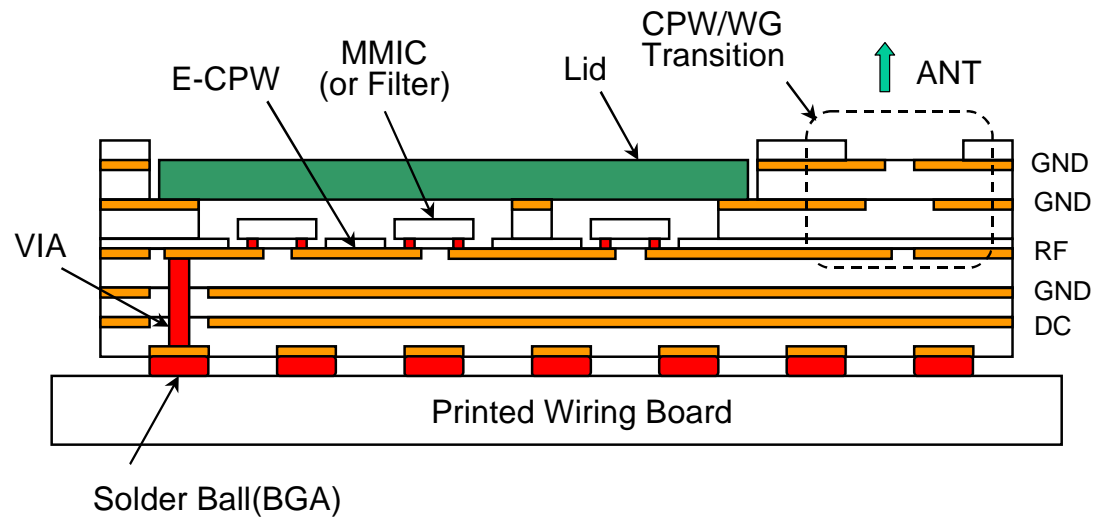

Fig. 2 Schematic cross-sectional view of the MCM. 


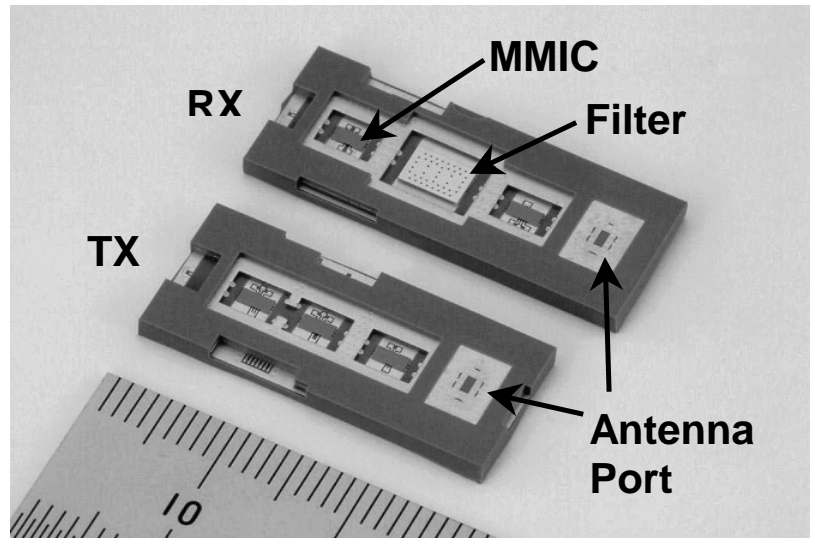

Fig.3 Photograph of the MCMs

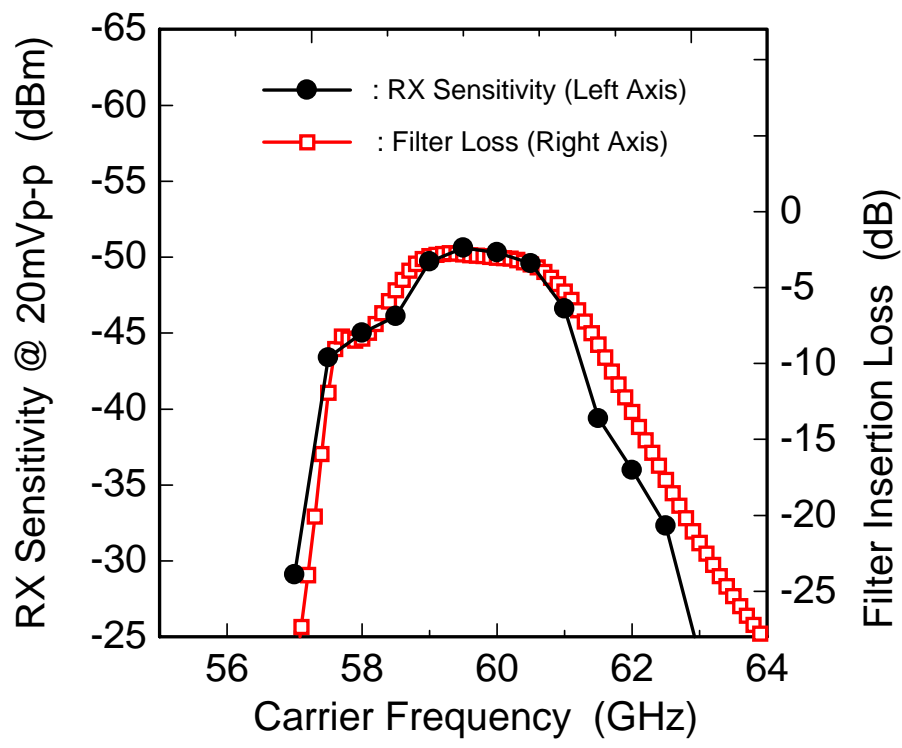

Fig. 5 RX frequency response for 500-Mbps ASK signal.

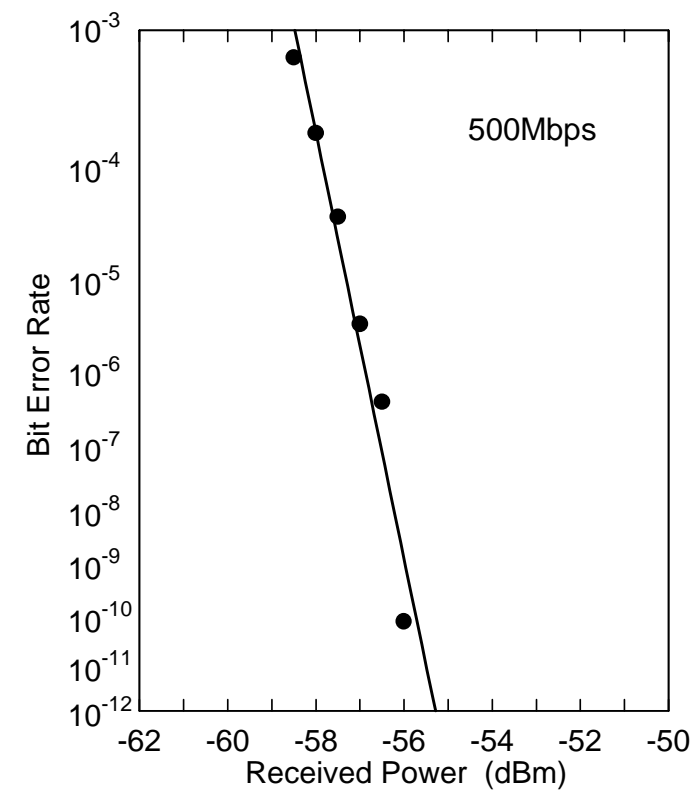

Fig. 7 BER performance of RX. $(60.05 \mathrm{GHz})$

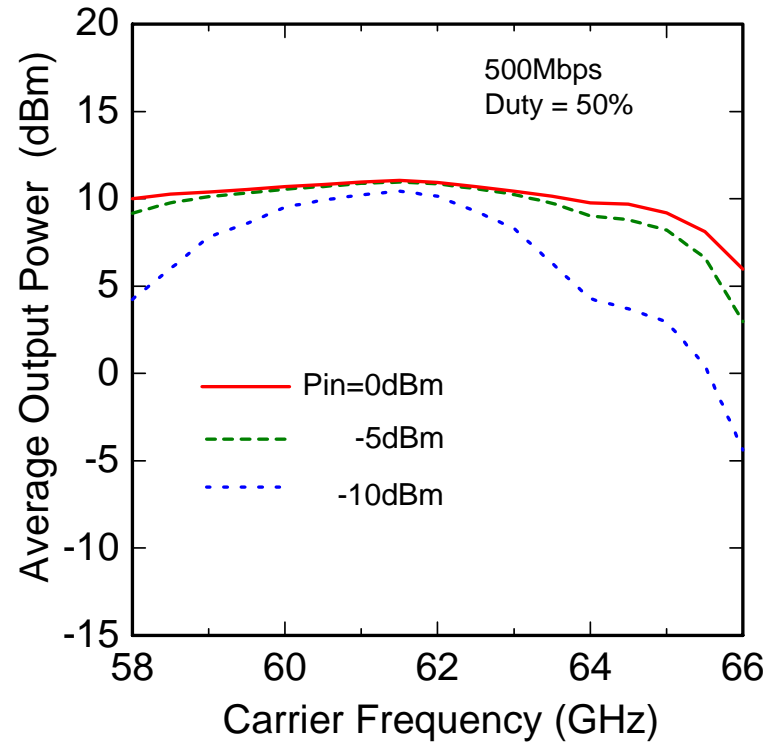

Fig.4 Average output power of TX.

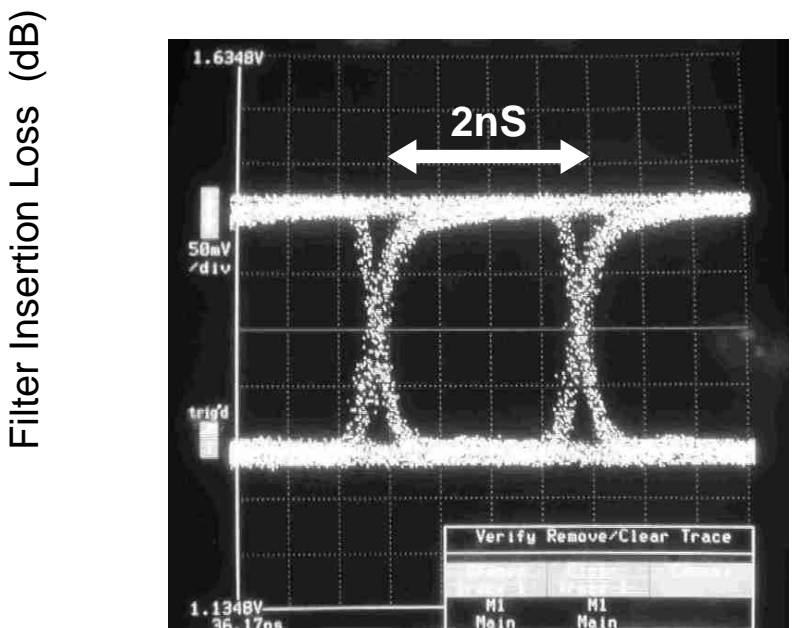

Fig. 6 Eye diagram of demodulated signal. (Received power: $-\mathbf{5 0} \mathrm{dBm}$ )

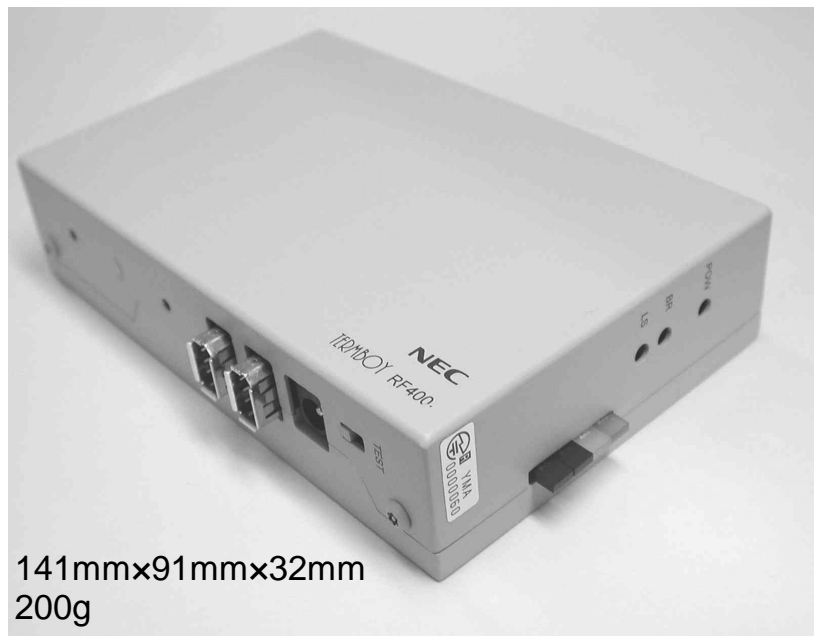

Fig. 8 IEEE1394 wireless adapter. 